\title{
28 Research Square \\ Infectious endocarditis: Improving diagnostic performance in native and prosthetic valve infection with 18F-FDG PET/CT
}

Ana Vera Marinho ( $\square$ ana.vera.marinho@gmail.com )

Centro Hospitalar e Universitario de Coimbra EPE https://orcid.org/0000-0003-3330-4943

José Paulo Almeida

Centro Hospitalar e Universitario de Coimbra EPE

Paula Soeiro

Centro Hospitalar e Universitario de Coimbra EPE

Rodolfo Silva

Centro Hospitalar e Universitario de Coimbra EPE

Francisco Gonçalves

Centro Hospitalar e Universitario de Coimbra EPE

Gracinda Costa

Centro Hospitalar e Universitario de Coimbra EPE

Lino Gonçalves

Centro Hospitalar e Universitario de Coimbra EPE

Maria João Ferreira

Centro Hospitalar e Universitario de Coimbra EPE

\section{Research article}

Keywords: infective endocarditis, 18F-FDG PET/CT, Modified Duke criteria, native valve endocarditis, prothesis valve endocarditis

Posted Date: December 5th, 2020

DOl: https://doi.org/10.21203/rs.3.rs-34293/v2

License: (c) (i) This work is licensed under a Creative Commons Attribution 4.0 International License.

Read Full License 


\section{Abstract}

Background: The diagnosis of infective endocarditis (IE) remains a clinical challenge. Diagnostic accuracy of the modified Duke criteria is suboptimal for native valve endocarditis (NVE) and even worse in the presence of prosthetic material-related infection (PVE). We aim to evaluate the diagnostic performance of 18F-FDG PET in patients with suspected IE referred to perform PET/CT.

Methods: Consecutive patients with suspected IE, referred to perform PET/CT between May 2016 and June 2019 were included. Diagnostic performance of modified Duke criteria (mDC) and PET/ CT for IE for NVE and PVE was determined.

Results: In total, 82 patients (mean age of $61 \pm 19$ years, $62 \%$ of male gender) were enrolled. There were 67 18F-FDG PET/CT concordant results with final diagnosis, corresponding to a $96 \%$ of agreement, $k=0.91(p=0.04)$. A SUV max cutoff value of $\geq 3.1$ identified positive cases with $88.9 \%$ sensitivity and $70.0 \%$ specificity. In patients with NVE, the addition of PET/CT to the $\mathrm{MDC}$ resulted in a reduction of the number of possible IE cases (from $58 \%$ to $4.3 \%$ ). In patients with PVE/intracardiac devices, PET/CT allowed reclassification of $67.4 \%$ of possible cases to $4.2 \%$. An alternative diagnosis was provided in $55.6 \%$ of the negative IE cases.

Conclusions: 18F-FDG PET/CT proved to be a useful diagnostic tool in patients with both suspected NVE and PVE with good sensitivity and specificity, resulting in a significant decrease of the number of possible endocarditis. Furthermore, it allowed the identification of the cause of clinical scenario in more than $50 \%$ of patients in which the diagnosis was excluded.

\section{Background}

The diagnosis of infective endocarditis (IE) is a clinical challenge, due to a myriad of nonspecific clinical presentations, involvement of different microorganisms and patient characteristics. Despite improvements in its both diagnostic procedures and management, IE remains associated with a poor prognosis, with an in-hospital mortality of $14-22 \%$ and 1-year mortality of $40 \%(1)$ (2). Modified Duke criteria, the cornerstone of its diagnosis, is largely based in the presence of positive blood cultures and evidence of endocardial involvement on echocardiography. The Duke criteria have been shown to be helpful for the diagnosis of NVE, with a sensitivity of $70-80 \%$, but lower values were found for prosthetic (PVE) and implantable cardiac electronic device (ICEDs) infections (3). In this population, clinical presentation is more frequently atypical, blood cultures negatives and diagnostic value of echocardiogram (TT and TOE) is lower and a negative result don't exclude the diagnosis, which leads to an erroneous diagnose in several patients (1) (4).

18F-FDG PET/CT, combining the high sensitivity of 18F-FDG PET to detect inflammation with the high spatial resolution of cardiac CT, is evolving as important additional method in difficult-to-diagnose cases. The main added value of this technique is the reduction in the rate of misdiagnosed IE, classified in the "possible IE category" using Duke criteria. Several reports have been demonstrated in detection of 
suspected PVE, CIED infections and its complications such as perivalvular extension and pulmonary emboli (5). Furthermore, whole-body 18F-FDG PET/CT has a power of detect a number of extracardiac metastatic foci in patients with known or suspected NVE or PVE which direct impacts in patient treatment (1)(6). An additional promising role include the monitoring response to antimicrobial treatment. In the most recent European Society of Cardiology (ESC) guidelines, 18F-FDG PET/CT has been included in the diagnostic algorithm PVE and also in the detection of embolic events for both NVE and PVE (7)(8). An interesting promising role yet insufficiently studied is detection of other extracardiac findings such as neoplastic lesions.

We aimed to evaluate the diagnostic performance of 18F-FDG PET in a cohort of patients with suspected IE referred to PET/CT in a tertiary care hospital.

\section{Methods}

\section{Study population}

A retrospective study was performed at a tertiary center with 18F-FDG PET/CT and included all referred patients for this exam for suspected IE between May 2016 and June 2019. The choice to perform 18FFDG PET/CT and the IE suspicion were based on the attending endocarditis team and did not follow a standardized protocol.

\section{Clinical, laboratory and microbiological data}

Baseline demographic characteristics of patients, including all relevant clinical data, were collected from hospital records at hospital admission. Laboratory data included basic renal and liver function test as well as hemogram. For microbiological data, all blood cultures with respective antibiotical sensitivity testing and, when applicable, serology, were recorded.

\section{Other imaging tests data}

Transthoracic echocardiography was performed in all patients and transesophageal echocardiography performed in $86.3 \%$, and recorded images and reports were analyzed. Data was extracted regarding assessments of general cardiac parameters, including cavity dimensions and ventricular function, and specific endocarditis suggestive findings such as valve function, vegetations (shape, size, and number), and periannular complications (abscess, pseudoaneurysm, perivalvular leak). 
Whole-body scans and dedicated cardiac bed images were obtained in a hybrid PET/CT scanner (Discovery ST, General ElectricsÒ). A [18F] FDG myocardial uptake suppression protocol, that included a 48h high-fat and low-carbohydrate diet and a fasting period of at least 12 hours, was used to optimize image quality and diagnostic accuracy. To further improve image quality $50 \mathrm{IU} / \mathrm{kg}$ of unfractionated heparin was administered, 15 minutes prior to radiotracer injection, in a few patients. Blood glucose levels were required to be less than $180 \mathrm{mg} / \mathrm{dL}$ during a period of approximately $60 \mathrm{~min}$ before the administration of the 18F-FDG.

Attenuation corrected images were used to locate and examine the hypermetabolic foci. However, nonattenuation corrected images were also reviewed, particularly to assess hypermetabolic foci near metallic structures, mainly valvular prosthesis, medical devices and electrodes. Images were reviewed by both a nuclear medicine physician and a cardiologist with several years of experience in cardiac [18F] FDG $\mathrm{PET} / \mathrm{CT}$.

Uptake intensity was measured by means of maximum Standardized Uptake Values (SUVmax) in patients with and without findings suggestive of infection. Uptake patterns were also noted. Cardiac and extra-cardiac findings were reported. Extra-cardiac findings included both those attributable to IE, such as embolic events, but also indicative of other pathologies, such as neoplasic lesions.

\section{Final diagnosis}

The final diagnosis of IE (gold standard) was established by consulting the final diagnosis attributed to the patient by the Endocarditis team at the time of hospital discharge or death, after being possession of clinical, microbiological, and imaging information as well as clinical response to treatment.

\section{Statistical analysis}

Continuous data are presented as the mean +/-SD. Categorical data are expressed as percentages. Sensitivity, specificity, positive and negative predictive values of DC at hospital admission, TOE echo and $\mathrm{PET} / \mathrm{CT}$, in evaluation of NVE and PVE were estimated. Receiver-operating characteristic (ROC) curves were built to determine the cutoff values with the best sensitivity-specificity combination to detect infection considering a binary outcome ( $1=$ positive $0=$ negative). Continuous data was tested for normality with the Shapiro-Wilks test and the Student's $t$ or the Mann-Whitney test was applied to compare them as appropriate. Fisher exact test was used for nominal variables to assess differences between groups. In these analyses we used the final diagnosis of confirmed and the rejected NVE and PVE as the gold standard outcome. P values of less than 0.05 were considered statistically significant. Statistical analyses were performed using SPSS (version 23.0; SPSS Inc.). 


\section{Results}

Clinical and microbiological data and echocardiography findings

In total, 82 patients (mean age of $61+/-19$ years, $62 \%$ of male gender) were enrolled from May 2016 to June 2019. Baseline characteristics were summarized in Table 1.

Half of included patients had an implanted prosthetic valve $(n=35)$, the majority of which biological $(n=19)$ and in aortic position $(n=27)$.

Fever was present in most patients $(n=64)$ (Table 2), followed by heart failure symptoms and vascular occurrences. One patient presented with pacemaker pocket signs of infection. Staphylococcus aureus was identified in 19 cases and more than half of these were methicillin resistant (52.9\%). Presence of vegetations was the most common echocardiographic finding $(n=31)$, followed by moderate to severe regurgitation $(n=14)$, presence of abscess, pseudoaneurysm $(n=9)$ or fistulae $(n=5)$.

\section{PET/CT findings}

Seventy patients $(90.9 \%)$ were under antibiotic therapy before PET/CT with a mean interval between treatment initiation and PET/ CT of $20 \pm 14$ days. PET/CT was positive for IE in 24 cases. The receiveroperating characteristic curve yielded an area under the curve of 0.91 (95\% confidence interval, 0.880.96 ) for SUVmax. A SUVmax cutoff value of $\geq 3.1$ identified positive cases with $88.9 \% \%$ sensitivity and $70.0 \%$ specificity (Figure 1).

\section{PET/ CT performance in IE of NVE and PVE diagnosis}

There were 6718F-FDG PET/CT concordant results with the final diagnosis of the endocarditis team, corresponding to a $96 \%$ of agreement, $k=0.91(p=0.04)$.

PET/CT was positive in 1 patient without a final diagnosis of IE (false positive, 2.2\%). Conversely, PET/CT was negative in 4 patients with a diagnosis IE (false negative, $5.7 \%$ ).

The sensitivity, specificity, positive, negative predictive values and area under the curve ( $95 \%$ confidence intervals) of DC at admission, echocardiogram and PET/CT in the diagnosis of IE are shown in Table 3.The addition of PET/CT to the modified DC resulted in a substantial increase in diagnostic specificity (from $33.3 \%$ to $97.8 \%$ ) with only a mild reduction in sensitivity (from $88.0 \%$ to $84.0 \%$ ). The percentage of IE cases classified as "possible" was reduced from $58 \%$ to $4.3 \%$.

Concerning the subgroup of patients with intracardiac devices, PET/CT showed similar sensitivity to DC at admission but superior to echocardiography ( $89.5 \%$ vs $68.4 \%$ ). PET/CT was also more specific than echocardiogram and DC at admission for diagnosis of device IE ( $90.0 \%$ vs $60.0 \%$ vs $51.0 \%)$. In this subgroup, PET/CT allowed reclassification, reducing from $67.4 \%$ of possible cases to $4.2 \%$. (table 4 ) 


\section{Extracardiac Findings: Additional Benefits of Whole-Body PET/CT}

PET/CT detected 4 cases of peripheral embolism, which were asymptomatic. In addition, PET/CT identified clinical important extracardiac findings in 34 patients, and provided an alternative diagnosis in 22 of the negative IE cases: other infection foci in 13 patients [pneumonia $(n=3), Q$ fever $(n=5)$, colon abscess/diverticulitis $(n=1)$, spondylodiscitis $(n=5)$, hepatic abscess $(n=2)$, phlebitis $(n=1)$, sternotomy infection $(n=1)$ ]; 1 patient with Takayasu's arteritis exacerbation and 8 unsuspected neoplastic lesions [colon $(n=1)$, kidney $(n=1)$, testicle $(n=1)$ and lymphoma $(n=5)]$, (figure $2-5)$.

\section{Final Diagnosis and Patient Management}

The patients were followed during a mean of $14+/-10$ months. According to diagnosis gold standard defined for this study, IE was established in 22 patients and was rejected in 45 patients; 3 patients were classified as possible IE. Those patients with definitive diagnosis of IE, 11 were submitted to surgery or percutaneous extraction of leads or devices. The remaining patients were medically managed. The allcause mortality was $17.1 \%$ (12 patients).

\section{Discussion}

Our study finds that the use of PET/CT adds further diagnostic information to classical DC in patients with suspected IE, particularly those with prosthetic valves and intracardiac devices. This may have a significant effect on the choice of an appropriate strategy and therefore impacts IE-related morbidity and mortality.

Infective endocarditis is more common now than in the past, with its incidence increasing from 9.3 per 100000 population in 1998 to 15 per 100000 in 2011(9). Partially this is due to health care-associated disease (10). In a large multicenter, multinational study, health care-associated infective endocarditis accounted for $34 \%$ of cases (11). Hemodialysis, non-hemodialysis intravascular catheters, and invasive procedures are often associated with the infection (12). Furthermore, the proportion of cases related with prosthetic valves and implantable cardiac devices is increasing(9). Despite major advances in diagnostic and therapeutic procedures, the prognosis is poor with a 1-year mortality approaching $30 \%$ and high complication rates at long term(1)(13).

Patients with intracardiac devices or prosthetic valves are challenging IE population because of frequent atypical presentation, different epidemiological profile (more prevalence of MRSA, fungi and multirresistent agents), and higher incidence of perivalvular extension and complications and increased mortality. The traditional modified Duke criteria are difficult to use in these patients due to the challenging interpretation of lesions on echocardiography and a negative study did not exclude the diagnosis. several cases of suspected IE are left without a conclusive diagnosis (2). The scenario is even more problematic in case of suspected ICEDs infection, in which patients frequently present with nonspecific clinical 
manifestations and both TT and TOE were limited in evaluation of cardiac right chambers.. Intracardiac echo was recently found to be feasible and effective and showed superior sensitivity for detection of vegetations in cardiac devices. However a complete normal echocardiographic examination does not rule out (14). Consequently, these patients may be recurrently hospitalized for an inflammatory/infectious disorder of unknown origin despite detailed investigations.

According ESC guidelines, complete hardware removal should be considered on the basis of occult infection, however in the absence of proven lead infection, further clinical management including the removal of the system is frequently postponed which can result in recurrences and significant mobility and mortality (3)(15).

The 2015 ESC Guidelines on Endocarditis recommend using additional imaging modalities when echocardiography and blood cultures are inconclusive (i.e. result in a 'possible' diagnosis of endocarditis, or a 'rejected' diagnosis with persisting high suspicion). Three techniques may be employed: CTA to depict perivalvular complications, cerebral magnetic resonance imaging (MRI), and/or whole-body SPECT/CT or PET/ CT to evaluate the presence of abnormal metabolic activity around the prosthetic valve and exclude embolic events (16).

In a cohort of patients with suspected IE referred to PET/CT in a tertiary care hospital the its diagnostic performance was evaluated. Comparing with modified DC the use of PET increased significatively the specificity for the diagnosis of IE from 33 to $98 \%$ in NVE and from 15.8 to $94.5 \%$ in PVE with similar sensitivity. In a metanalysis, Maryam Mahmood et al including 13 studies involving 573 patients showed, a pooled sensitivity of PET/CT for diagnosis of IE of $76.8 \%$ and the pooled specificity was $77.9 \%$. Diagnostic accuracy was improved for PVE with sensitivity of $80.5 \%$ and specificity of $73.1 \%$ (17). However, some of these studies included initially rejected patients by modified Duke criteria, probably making less cost-effective the indication of 18F-FDG PET/CT in suspected IE (6). Our study did not include initially rejected IE patients by modified Duke criteria showing a high specificity in all the indications in which 18F-FDG PET/CT was performed. Furthermore, the results of this study indicate that substantial benefits can be obtained by including PET/CT in the diagnostic workup of patients with both native and with prosthetic valves/ intracardiac devices suspected IE.

No distinction between biological and mechanical prosthetic valves was made in our study. Roque et al., in a study evaluating metabolic patterns of captation after surgery, no differences between biological or mechanical prothesis were found(18). Evidence of its use in patients suspected of transcatheter-replaced aortic valves (TAVR) endocarditis is still limited to case reports, but the exponential growing rates of TAVR implantation in last years coupled with its implantion in older population with several comorbilities, increasing rates of TAVR endocarditis were expected. In our cohort, 2 patients had TAVR endocarditis suspicion and 1 patient had a previous "mitraclip" implantation. In all cases, PET-FDG excluded the diagnosis.

The 4 false-negative cases, in our study, could be due previous antibiotherapy before PET/CT; and presence of small lesions below the metabolic/spatial resolution of PET/CT. In fact, both false negative 
and false positive results have been reported in previous studies. False negative results might be due to prior administration of antimicrobial therapy, small size of vegetations, and elevated blood glucose concentration. False positive results might be a result of recent cardiac procedures and inadequate patient preparation(19).

Even when criteria for definitive IE category diagnosis was established by use Modified Duke criteria, there are other valid reasons to consider both PET/CT which include: evaluation of involvement of other valves or cardiac implanted electronic devices, identification of port of entry, identification of other foci of infection or other cause for clinical picture, all of which may change the treatment strategy(1). The presence of septic emboli is crucial for the correct management of patients with IE. Asymptomatic metastatic foci is present has been identified in up $20 \%$ to $50 \%$ of patients, mainly spleen (15),(20),(21). Failure to identify metastatic infection complications may lead to early interruption of therapy, thus potentially triggering relapse and an unfavorable outcome (22). In our cohort, PET/CT identified cases of clinically unsuspected septic embolism and clinical important extracardiac findings. It also provided an alternative or additional diagnosis in more than half of the negative cases, including 13 cases of neoplasm previous unknown.

\section{Limitations}

This was a single-center study with recognized limitations. Our sample size was insufficient to allow robust subgroup analyses such as the performance of PET/CT in mechanical vs biological prothesis or intracardiac devices vs prothesis. The gold standard for the diagnosis was the clinical judgment of the endocarditis team based on the results of diagnostic tests and the clinical follow-up of patient. Finally, in our study the time between the beginning of antibiotherapy and the PET/CT was longer than reported by other studies.

\section{Conclusions}

18F-FDG PET/CT proved to be a useful diagnostic tool in patients with both suspected NVE and PVE with good sensitivity and excellent specificity, resulting in a significant decrease in the number of cases of possible El. This significant diagnostic improvement, which could have an important impact on IE outcomes, merits further assessment in larger series. In particular the clinical impact of occult cancer diagnosis should be addressed.

\section{Abbreviations}

18F-FDG PET/CT: 18F-fluorodeoxyglucose positron emission tomography/computed tomography

IE: infective endocarditis 
ICED: implantable cardiac electronic device

mDK: Modified Duke criteria

NVE: native valve endocarditis

PVE: prosthetic material-related infection

TTE: transthoracic echocardiography

TEE: transoesophageal echocardiography

SUVmax: maximum Standardized Uptake Values

\section{Declarations}

Authors information

Affiliations

1. Departament of Cardiology, Centro Hospitalar e Universitário de Coimbra, Praceta Prof. Mota Pinto, Coimbra, 3000-075, Portugal

Ana Vera Marinho, MD; José Paulo Almeida MD; Francisco Gonçalves MD; Lino Gonçalves, PhD; Maria João Ferreira, $\mathrm{PhD}$

2. Department of Nuclear Medicine, Centro Hospitalar e Universitário de Coimbra, Praceta Prof. Mota Pinto, Coimbra, 3000-075, Portugal

Paula Soeiro MD; Rodolfo Silva MD; Gracinda Costa MD.

3. Institute of Nuclear Sciences applied to Heath (ICNAS), Coimbra University. Coimbra, Portugal Rodolfo Silva MD; Maria João Ferreira, PhD

4. Faculty of Medicine of Coimbra University. Coimbra, Portugal Lino Gonçalves, PhD; Maria João Ferreira, PhD 
Corresponding author:

Ana Vera Marinho, MD

\section{Ethics approval and consent to participate}

A written consent of all participating patients was obtained. The study was carried out according to the principles of the Declaration of Helsinki and approved by the local ethics committees- Health's Ethics Committee of Centro Hospitalar e Universitário de Coimbra / Faculdade de Medicina da Universidade de Coimbra.

\section{Consent for publication}

Not applicable

\section{Availability of data and materials}

The dataset generated during and analyzed during the current study is available from the corresponding author on reasonable request.

\section{Competing interest}

The authors don't have any potential conflict of interest

\section{Fundings}

No fundings to declare

\section{Author Contributions Statement}

AVM, RS and MJF conceived the study and designed the trial. AVM, JA and PS collected information. AVM, JA, GC and MJF provided statistical advice on study design and analyzed the data. AVM drafted the manuscript FG, LG, MJF reviewed the manuscript. All authors reviewed the final manuscript and consent publication. 
Acknowledgements

The authors would like to thank the ICNAS staff of our hospital and all referring physicians

\section{References}

1. Gomes A, Glaudemans AWJM, Touw DJ, van Melle JP, Willems TP, Maass AH, et al. Diagnostic value of imaging in infective endocarditis: a systematic review. Lancet Infect Dis [Internet]. 2017;17(1):e114. Available from: http://dx.doi.org/10.1016/S1473-3099(16)30141-4

2. Lancellotti P, Habib G, Oury C, Nchimi A. Positron Emission Tomography/Computed Tomography Imaging in Device Infective Endocarditis: Ready for Prime Time. Circulation. 2015;132(12):1076-80.

3. Habib G, Lancellotti P, Antunes MJ, Bongiorni MG, Casalta JP, Del Zotti F, et al. 2015 ESC Guidelines for the management of infective endocarditis. Vol. 36, European Heart Journal. 2015. 3075-3123 p.

4. Hill EE, Herijgers P, Claus P, Vanderschueren S, Peetermans WE, Herregods MC. Abscess in infective endocarditis: The value of transesophageal echocardiography and outcome. A 5-year study. Am Heart J. 2007;

5. Fowler VG, Miro JM, Hoen B, Cabell CH, Abrutyn E, Rubinstein E, et al. Staphylococcus aureus endocarditis: A consequence of medical progress. J Am Med Assoc. 2005;

6. Pizzi MN, Roque A, Fernández-Hidalgo N, Cuéllar-Calabria H, Ferreira-González I, Gonzàlez-Alujas MT, et al. Improving the Diagnosis of Infective Endocarditis in Prosthetic Valves and Intracardiac Devices with 18F-Fluordeoxyglucose Positron Emission Tomography/Computed Tomography Angiography: Initial Results at an Infective Endocarditis Referral Center. Circulation. 2015;132(12):1113-26.

7. Habib G, Lancellotti P, Antunes MJ, Bongiorni MG, Casalta JP, Del Zotti F, et al. 2015 ESC Guidelines for the management of infective endocarditis: The Task Force for the Management of Infective Endocarditis of the European Society of Cardiology (ESC). Eur Heart J. 2015;

8. Millar BC, de Camargo RA, Alavi A, Moore JE. PET/Computed Tomography Evaluation of Infection of the Heart. PET Clin. 2019;

9. Wang A, Gaca JG, Chu VH. Management Considerations in Infective Endocarditis A Review. 2018; (March).

10. Toyoda N, Chikwe J, Itagaki S, Gelijns AC, Adams DH, Egorova NN. Trends in infective endocarditis in California and New York state, 1998-2013. JAMA - J Am Med Assoc. 2017;

11. Almirante B, Tornos P, Pigrau C, Sambola A, Igual A, Pahissa A. Contemporary epidemiology and prognosis of health care-associated infective endocarditis. Chinese J Infect Chemother. 2009;9(4):296.

12. Delahaye F, M'Hammedi A, Guerpillon B, De Gevigney G, Boibieux A, Dauwalder O, et al. Systematic Search for Present and Potential Portals of Entry for Infective Endocarditis. J Am Coll Cardiol. 2016;

13. Thuny F, Giorgi R, Habachi R, Ansaldi S, Le Dolley Y, Casalta JP, et al. Excess mortality and morbidity in patients surviving infective endocarditis. Am Heart J. 2012; 
14. Bongiorni MG, Di Cori A, Soldati E, Zucchelli G, Arena G, Segreti L, et al. Intracardiac echocardiography in patients with pacing and defibrillating leads: A feasibility study. Echocardiography. 2008;25(6):632-8.

15. Granados U, Fuster D, Pericas JM, Llopis JL, Ninot S, Quintana E, et al. Diagnostic accuracy of 18FFDG PET/CT in infective endocarditis and implantable cardiac electronic device infection: A crosssectional study. J Nucl Med. 2016;57(11):1726-32.

16. Habib G, Lancellotti P, Antunes MJ, Bongiorni MG, Casalta JP, Del Zotti F, et al. Ghid de management al endocarditei infecţioase 2015. Vol. 26, Revista Romana de Cardiologie. 2016. 343-405 p.

17. Mahmood M, Kendi AT, Ajmal S, Farid S, O'Horo JC, Chareonthaitawee P, et al. Meta-analysis of 18FFDG PET/CT in the diagnosis of infective endocarditis. J Nucl Cardiol. 2019;26(3):922-35.

18. Roque A, Pizzi MN, Fernandez-Hidalgo N, Cuellar-Calabria H, Rios R, Ferreira N, et al. 524918F-FDG$\mathrm{PET} / \mathrm{CTA}$ of prosthetic cardiac valves: postsurgical inflammatory patterns and its temporal evolution. Can we question the 3-month limit of the current guidelines? Eur Heart J. 2018;39(suppl_1):2019.

19. Swart LE, Scholtens AM, Tanis W, Nieman K, Bogers AJJC, Verzijlbergen FJ, et al. 18Ffluorodeoxyglucose positron emission/computed tomography and computed tomography angiography in prosthetic heart valve endocarditis: From guidelines to clinical practice. Vol. 39, European Heart Journal. 2018. p. 3739-49.

20. Kestler M, Muñoz P, Rodríguez-Créixems M, Rotger A, Jimenez-Requena F, Mari A, et al. Role of 18FFDG PET in patients with infectious endocarditis. J Nucl Med. 2014;

21. Orvin K, Goldberg E, Bernstine H, Groshar D, Sagie A, Kornowski R, et al. The role of FDG-PET/CT imaging in early detection of extra-cardiac complications of infective endocarditis. Clin Microbiol Infect [Internet]. 2015;21(1):69-76. Available from: http://dx.doi.org/10.1016/j.cmi.2014.08.012

22. Shrivastav R, Ludhwani D, Kallur KR, Perimbeti S, Bandyopadhyay D, Jimenez RCO, et al. NATIONWIDE TRENDS IN COMPLICATIONS OF INFECTIVE ENDOCARDITIS IN UNITED STATES FROM 1999 TO 2014. J Am Coll Cardiol. 2019;

\section{Tables}

Table 1. Baseline characteristics of study population 


\begin{tabular}{|c|c|}
\hline Baseline Characteristic & Total $(n=82)$ \\
\hline Age (years) & $61 \pm 19$ \\
\hline Male gender & $51(62.2 \%)$ \\
\hline \multicolumn{2}{|l|}{ Symptoms } \\
\hline - $\quad$ Fever & $64(83.1 \%)$ \\
\hline - $\quad$ Heart failure symptoms & $11(14.9 \%)$ \\
\hline - Vascular phenomena & $9(9.00 \%)$ \\
\hline - Pocket infection signs & $1(1.3 \%)$ \\
\hline \multicolumn{2}{|l|}{ Past medical history: } \\
\hline - Hypertension & $50(63.3 \%)$ \\
\hline - $\quad$ Diabetes & $17(21.8 \%)$ \\
\hline Cardiac Prosthesis/ Intracardiac device & $48(58.5 \%)$ \\
\hline Prosthesis type & $35(42.7 \%)$ \\
\hline - $\quad$ Bioprosthetic valve & $19(50.0 \%)$ \\
\hline - Mechanical valve & $12(31.6 \%)$ \\
\hline - $\quad$ Prosthesic ring & $4(10.5 \%)$ \\
\hline - $\quad$ Mitra clip & $1(2.60 \%)$ \\
\hline \multicolumn{2}{|l|}{ Localization } \\
\hline - $\quad$ Aortic & $27(69.2)$ \\
\hline - $\quad$ Mitral & $7(17.9 \%)$ \\
\hline - $\quad$ Aortic + mitral & $1(2.6 \%)$ \\
\hline - $\quad$ Pulmonar & $1(2.6 \%)$ \\
\hline Time from implantation to PET (years) & $4.7 \pm 6.6$ \\
\hline Intracardiac Devices & $18(22.0 \%)$ \\
\hline - $\quad$ Pacemaker & $13(59.1 \%)$ \\
\hline - $\quad I C D / C R T$ & $2(9.10 \%)$ \\
\hline - $\quad$ Dacron tube & $4(18.2 \%)$ \\
\hline - Patch & $1(4.5 \%)$ \\
\hline
\end{tabular}


Table 2. Echocardiography, laboratory and PET/CT findings

\begin{tabular}{|ll|}
\hline TT/ TOE Echocardiogram & $80(97.6 \%)$ \\
\hline - Moderate to severe regurgitation & $14(18.7 \%)$ \\
\hline - Vegetations & $31(39.7 \%)$ \\
\hline - Abcess/Pseudoaneurism & $9(11.8 \%)$ \\
\hline - Fistule & $5(6.6 \%)$ \\
\hline C-reactive protein (mg/dl) & $14.8 \pm 19$ \\
\hline White blood cells x10E9/L & $10.3 \pm 5.0$ \\
\hline Blood cultures /serology & $75(91.5 \%)$ \\
\hline - St aureurs & $19(43.2 \%)$ \\
\hline - Coagulase negative Staphylococci & $3(6.8 \%)$ \\
\hline - Oral streptococci & $2(4.5 \%)$ \\
\hline - Streptococcus bovis group & $8(18.2 \%)$ \\
\hline - Enterococcus spp. & $2(4.5 \%)$ \\
\hline - Gram negative bacilli & $2(4.5 \%)$ \\
\hline - Coxiella burnetii & $5(11.4 \%)$ \\
\hline Modified Duke criteria at admission & \\
\hline - Definitive & $13(16.9 \%)$ \\
\hline - Possible & $45(58.4 \%)$ \\
\hline - Rejected & $19(24.7 \%)$ \\
\hline Metabolic activity (PET/CT) & \\
\hline - SUV max & $3.3 \pm 1.4$ \\
\hline Peripheral embolism & $4(4.90 \%)$ \\
\hline Extracardiac findings & $34(41.0 \%)$ \\
\hline
\end{tabular}


Table 3. Diagnostic Performance of the modified Duke criteria at admission, and PET/CT for native valve endocarditis

\begin{tabular}{|llllll|}
\hline & Sensitivity & Specificity & PPV & NPV & AUC \\
\hline Modified DuKe Criteria & 88.0 & $33.3 \%$ & $33.3 \%$ & $33.3 \%$ & 0.60 \\
& {$[(38.8-69.6)$} & {$[86.1-91.7]$} & {$[86.1-91.7]$} & {$[86.1-91.7]$} & $(0.46-0.74)$ \\
\multirow{2}{*}{ PET/CT } & $84.0 \% \%$ & $97.8 \%$ & $87.5 \%$ & $83.3 \%$ & 0.83 \\
& {$[71.5-100.0]$} & {$[52.4-63.1]$} & {$[3.5-12.2]$} & {$[98.2-100.0]$} & $(0.71-0.93)$ \\
\hline
\end{tabular}

Table 4. Diagnostic Performance of the modified Duke criteria at admission and PET/CT for prosthesis and intracardiac device endocarditis

\begin{tabular}{|llllll|}
\hline & Sensitivity & Specificity & PPV & NPV & AUC \\
\hline Modified Duke Criteria & 89.5 & $15.8 \%$ & $51.5 \%$ & $51.0 \%$ & 0.52 \\
& {$[38.8-69.6]$} & {$[86.1-91.7]$} & {$[23.4-44.5]$} & {$[97.5-99.6]$} & $(0.32-0.72)$ \\
\hline PET/CT & $89.5 \% \%$ & $94.7 \%$ & $94 \%$ & $90 \%$ & $0.85(0.70-0.97)$ \\
& {$[71.5-100.0]$} & {$[52.4-63.1]$} & {$[3.5-12.2]$} & {$[98.2-100.0]$} & \\
\hline
\end{tabular}

\section{Figures}




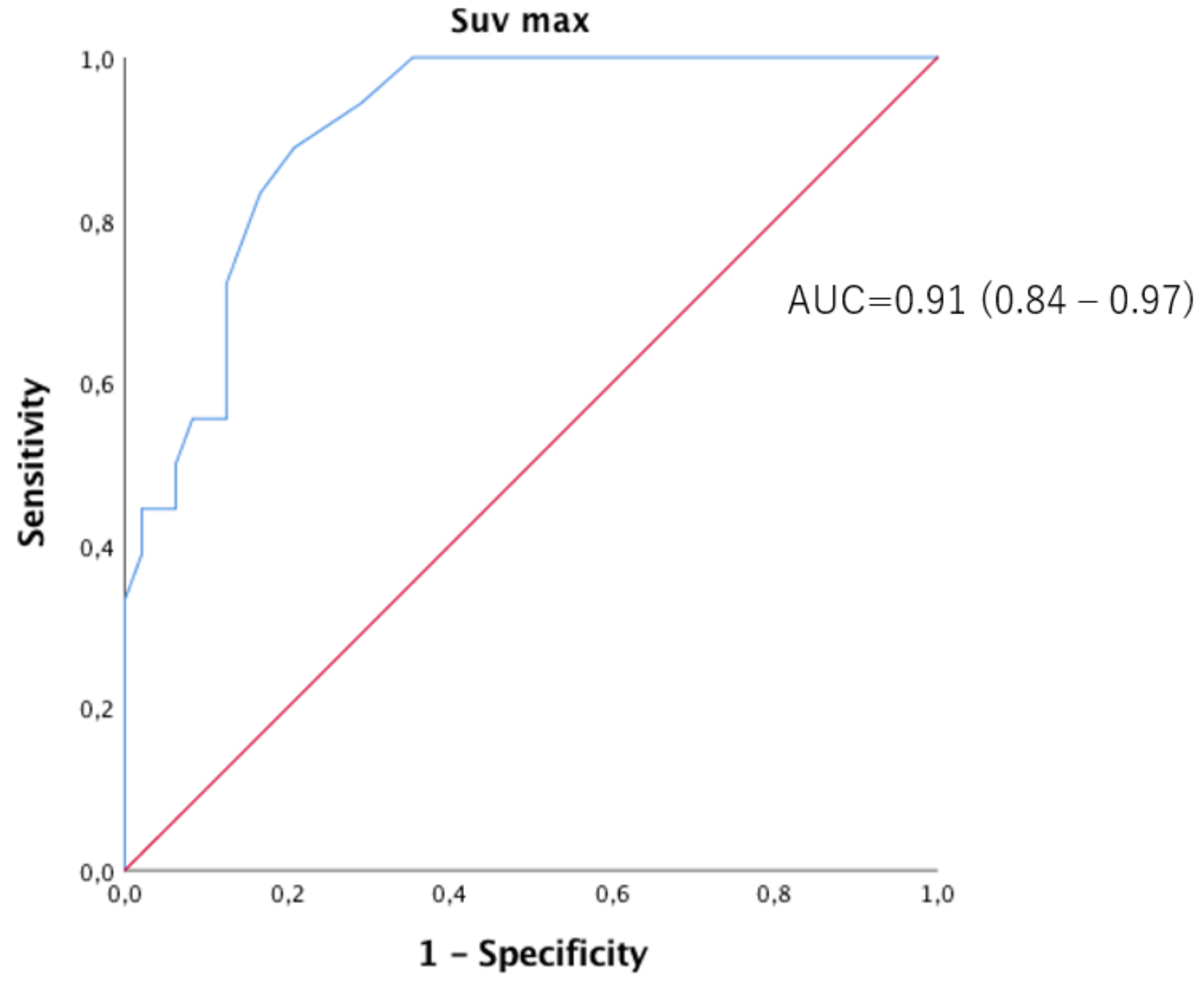

\section{Figure 1}

Maximum standardized uptake value (SUV max) receiver-operating characteristics (ROC) curve. A SUV max cutoff value of $\geq 3.1$ identified positive cases with sensitivity of $88.9 \%$ and $70.0 \%$ of specificity. 


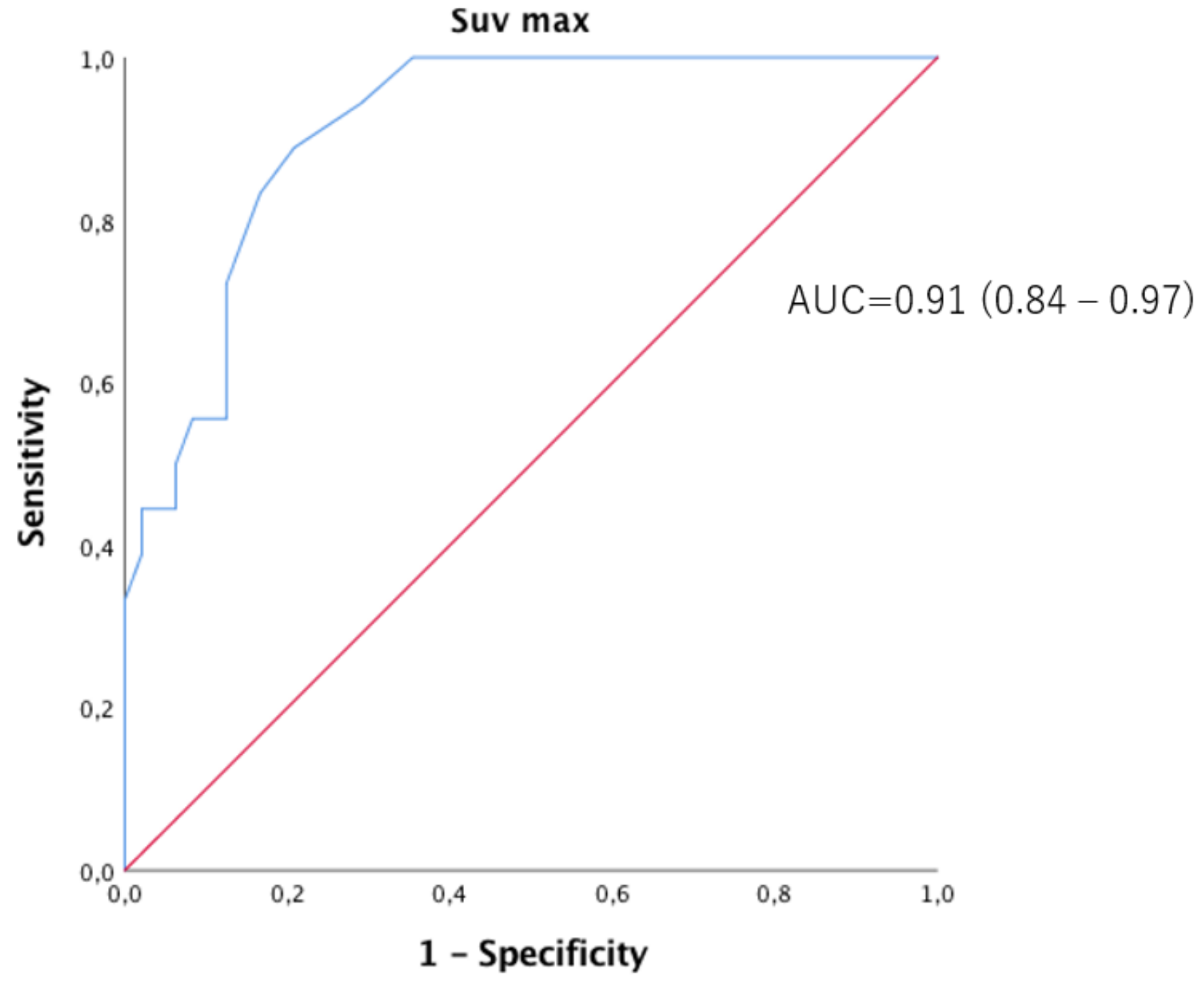

\section{Figure 1}

Maximum standardized uptake value (SUV max) receiver-operating characteristics (ROC) curve. A SUV max cutoff value of $\geq 3.1$ identified positive cases with sensitivity of $88.9 \%$ and $70.0 \%$ of specificity. 


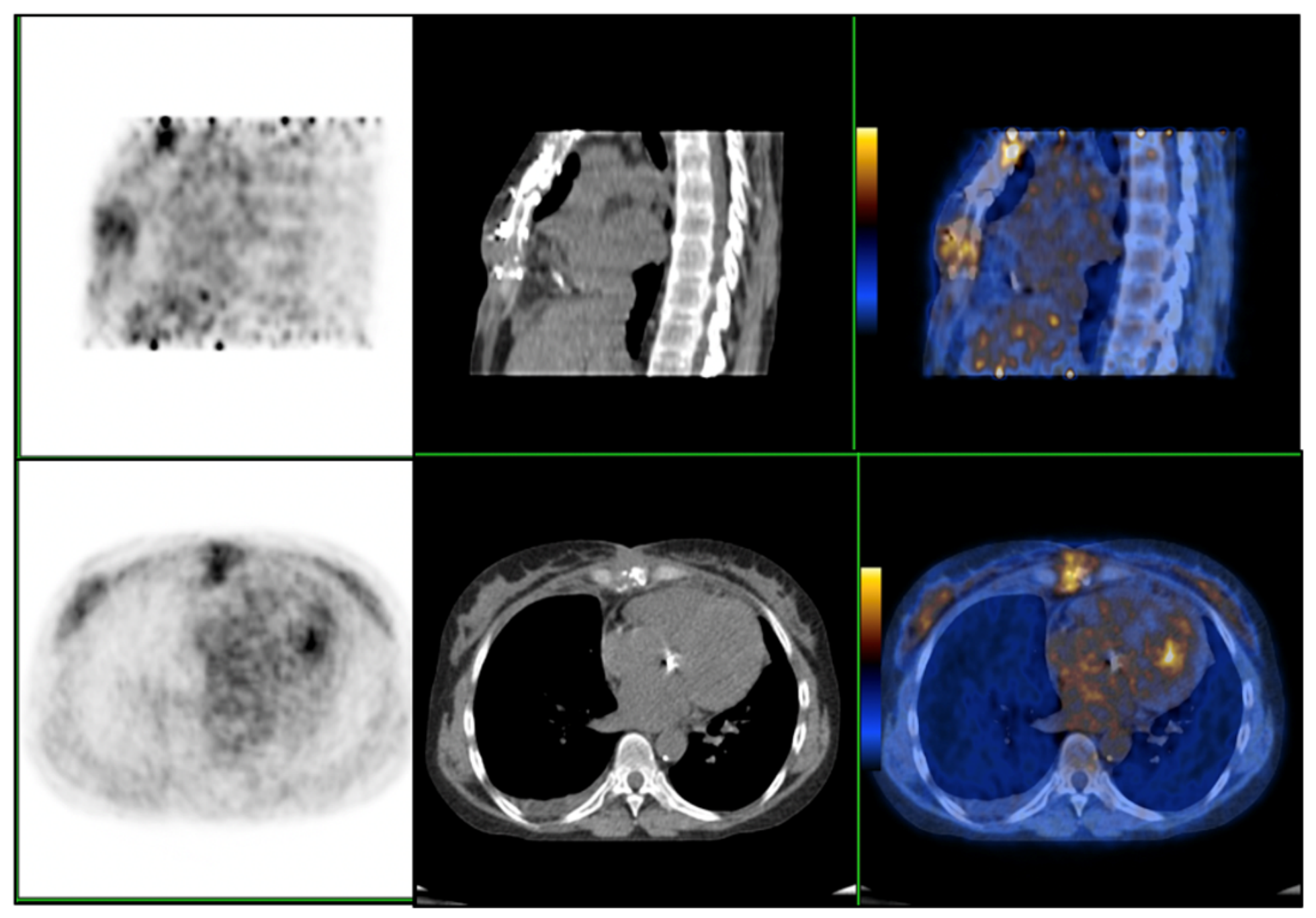

Figure 2

18F-FDG-PET/CT Scanning Identifying inflammation in local of sternotomy 


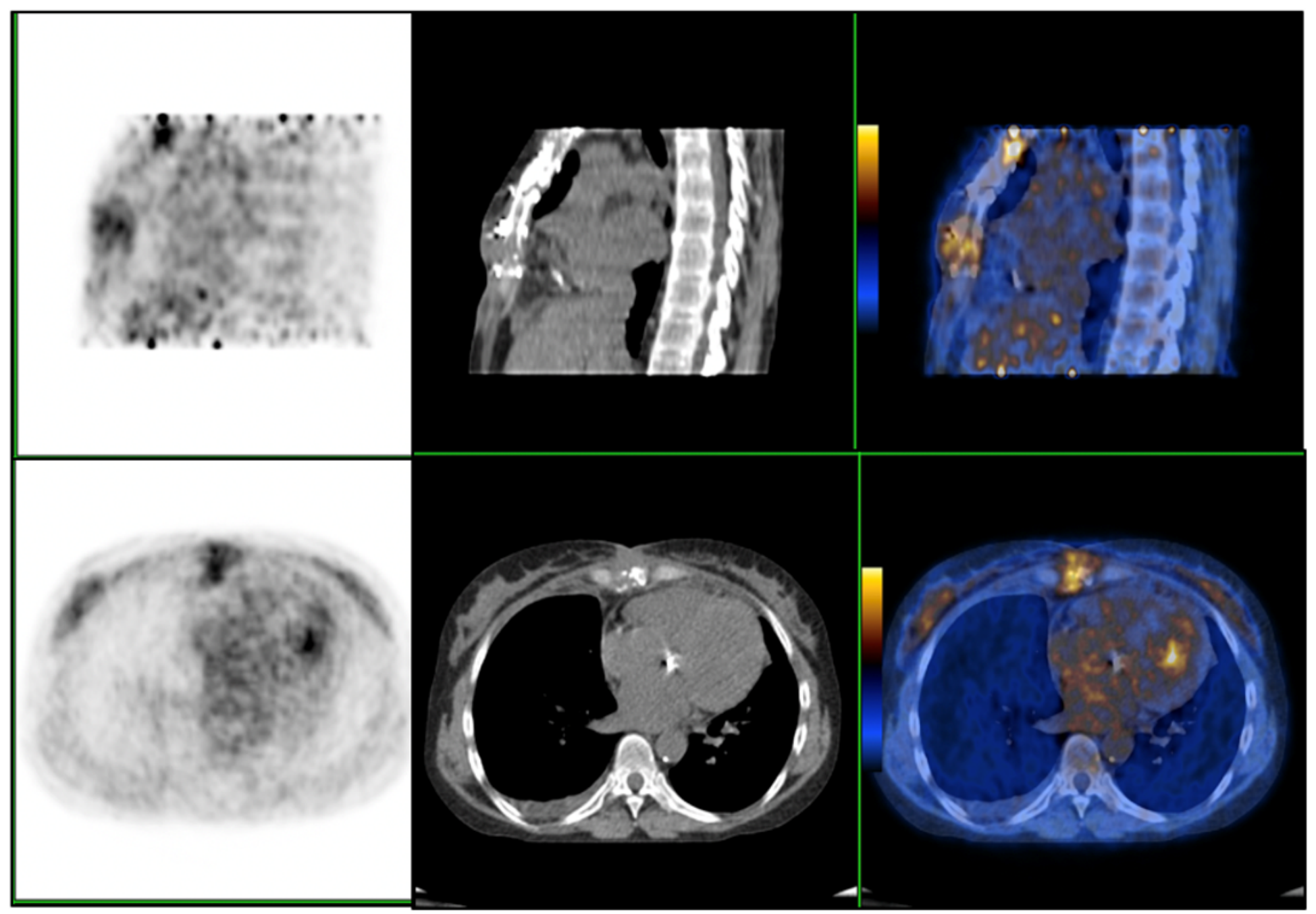

Figure 2

18F-FDG-PET/CT Scanning Identifying inflammation in local of sternotomy

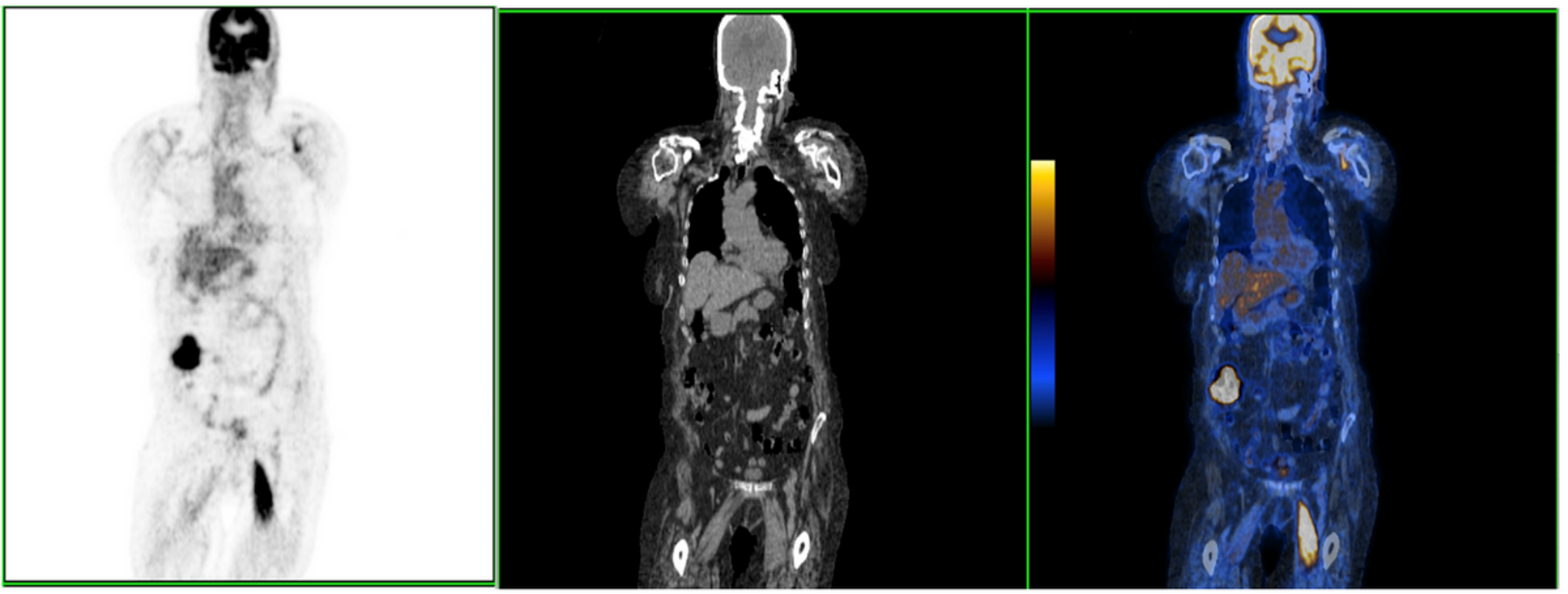

Figure 3 
18F-FDG-PET/CT Scanning Identifying a mass in ascending colon with high metabolic activity

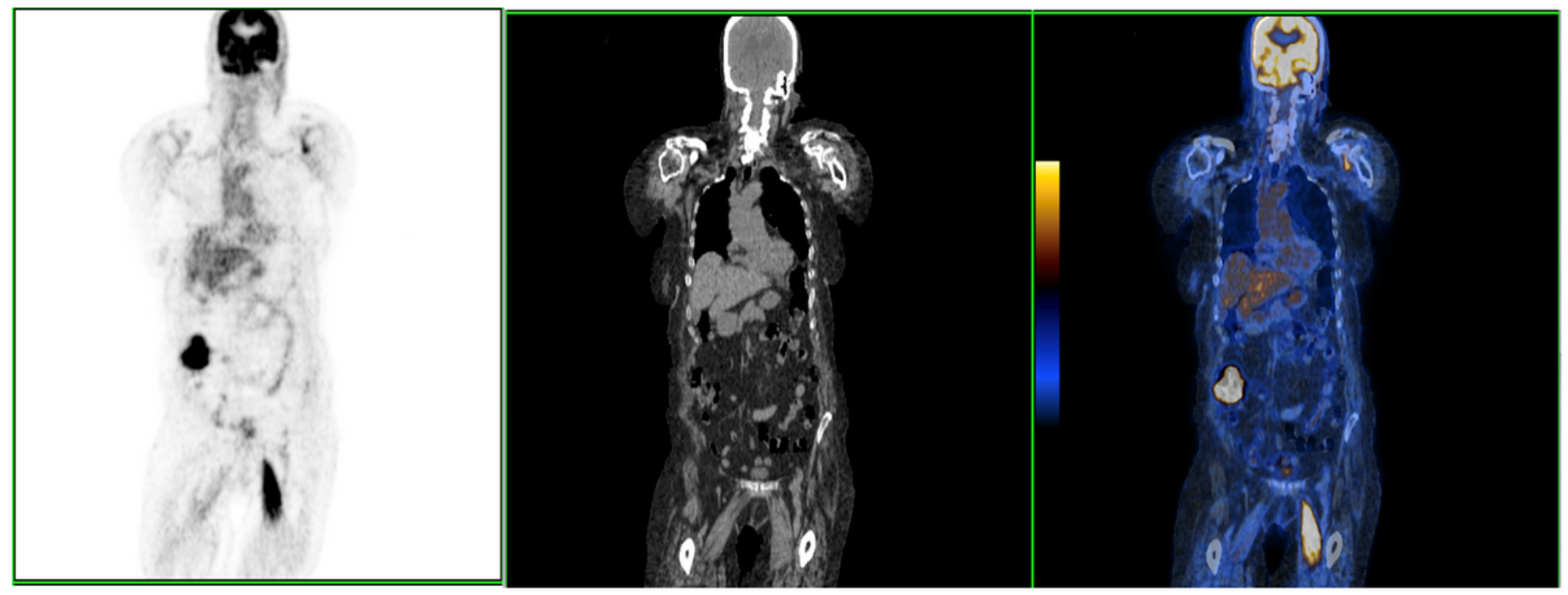

Figure 3

18F-FDG-PET/CT Scanning Identifying a mass in ascending colon with high metabolic activity

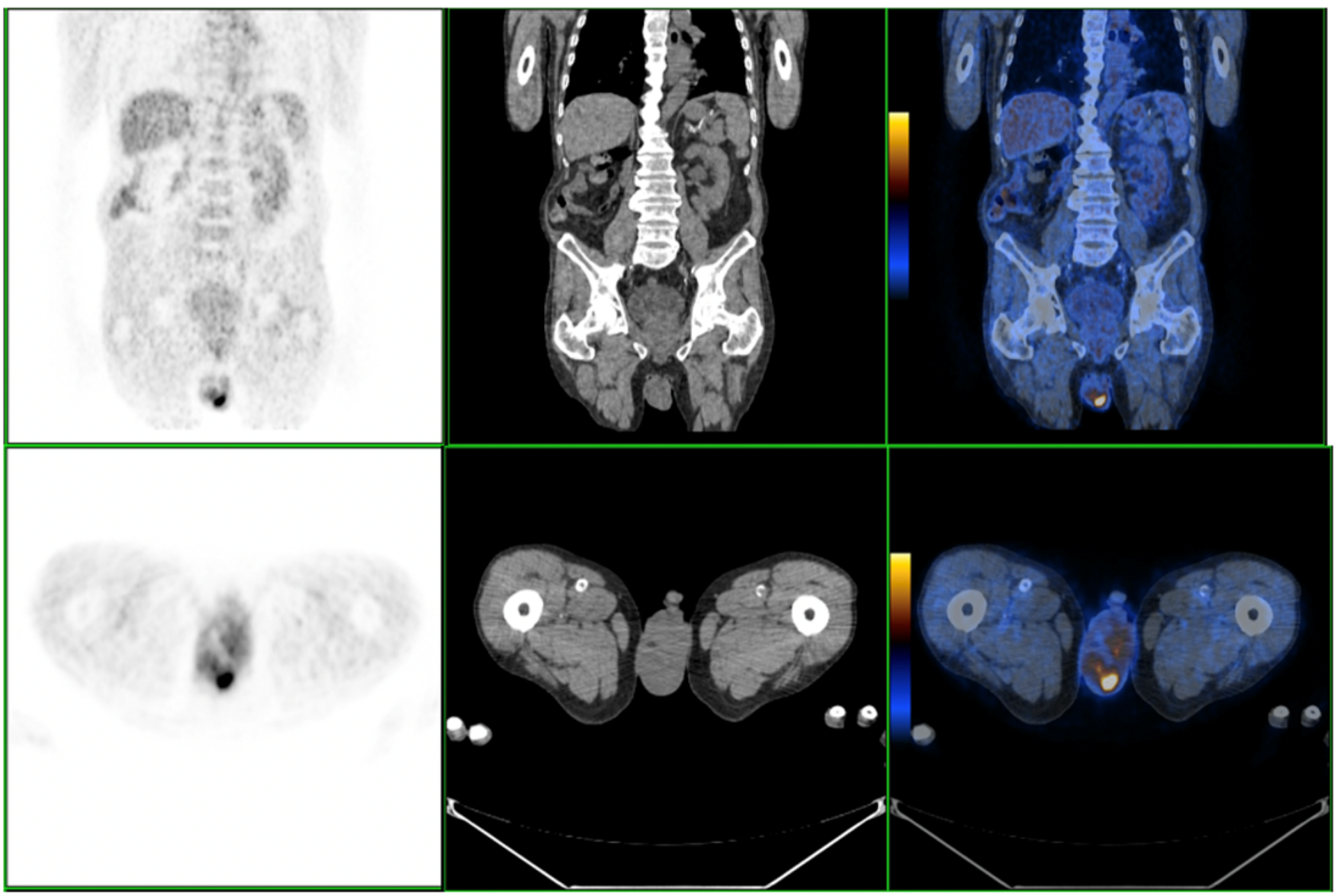

Figure 4 
18F-FDG-PET/CT Scanning Identifying a mass in testicle

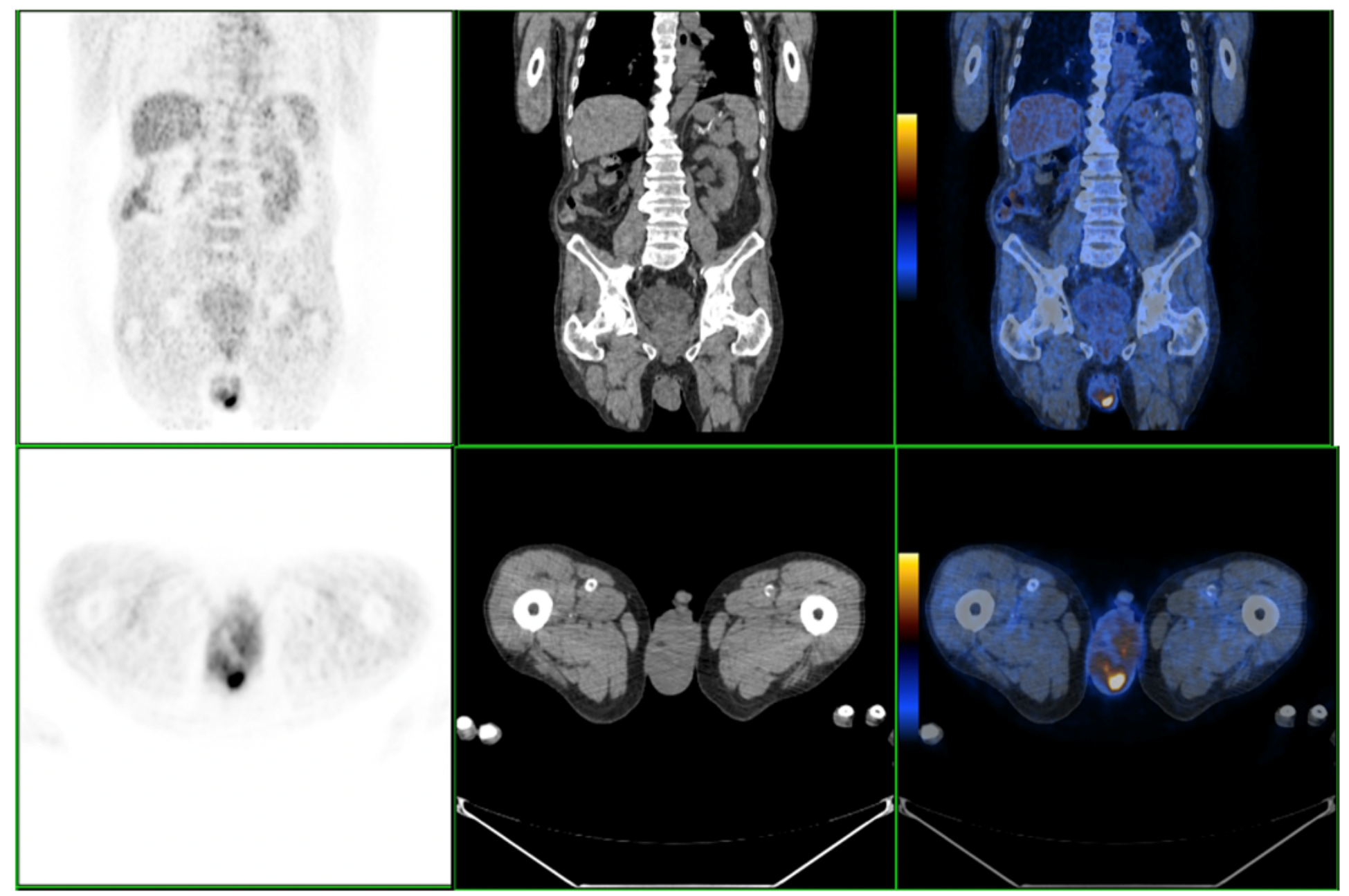

Figure 4

18F-FDG-PET/CT Scanning Identifying a mass in testicle 


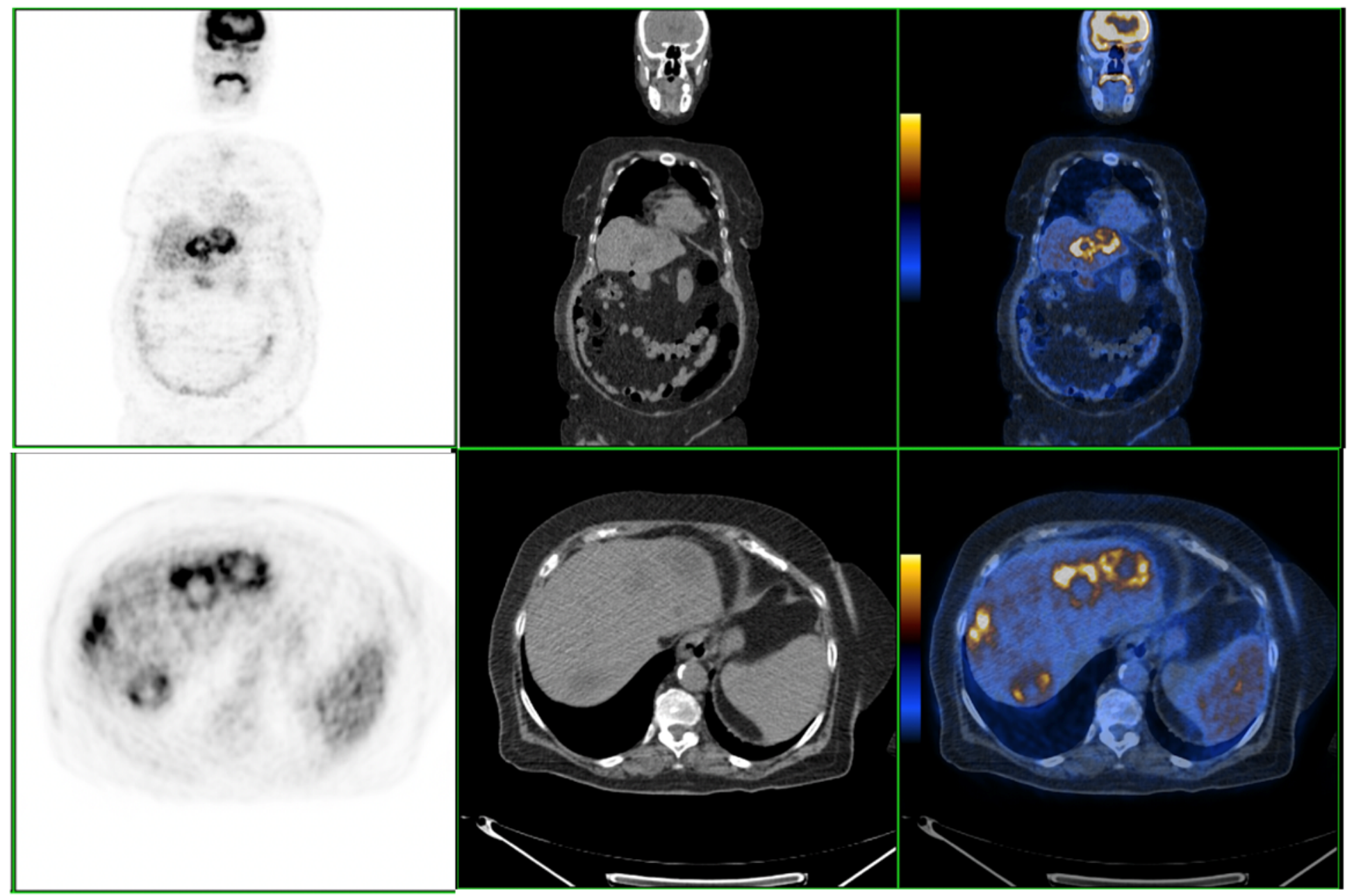

Figure 5

18F-FDG-PET/CT Scanning Identifying a hepatic abscess. 


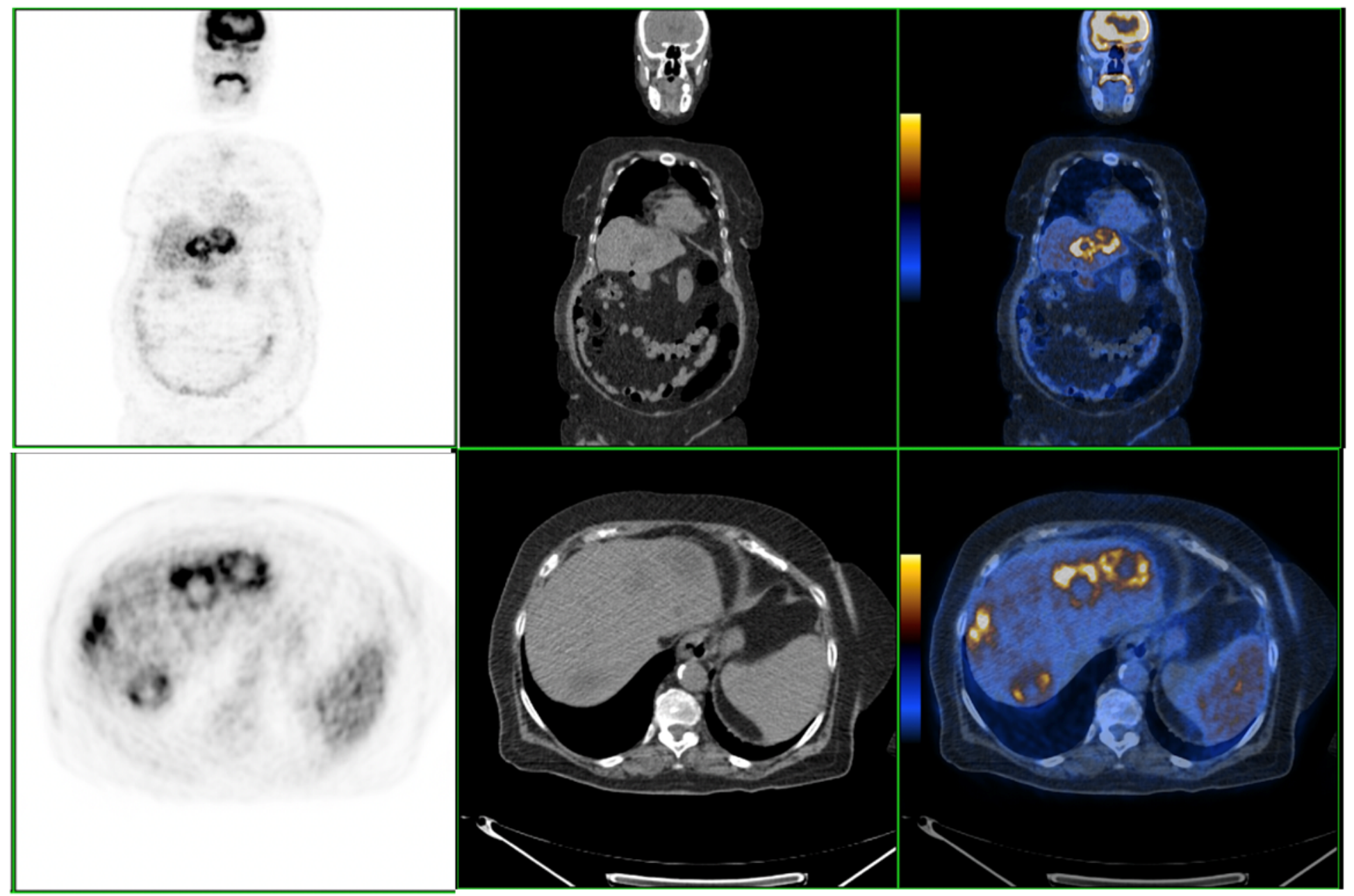

Figure 5

18F-FDG-PET/CT Scanning Identifying a hepatic abscess. 